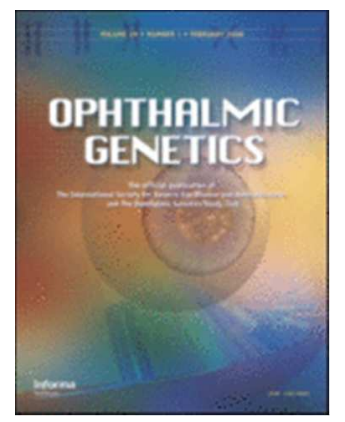

\title{
Retinitis pigmentosa and bilateral cystoid macular oedema in a patient heterozygous for the RIM1 mutation previously associated with cone-rod dystrophy 7
}

\begin{tabular}{|r|l|}
\hline Journal: & Ophthalmic Genetics \\
\hline Manuscript ID & NOPG-2015-0153.R3 \\
\hline Manuscript Type: & Case Report \\
\hline Complete List of Authors: & $\begin{array}{l}\text { Warwick, Alasdair; University Hospital Southampton NHS Foundation Trust, } \\
\text { Ophthalmology } \\
\text { Shawkat, Fatima; University Hospital Southampton NHS Foundation Trust, } \\
\text { Southampton Eye Unit } \\
\text { lotery, andrew; Southampton university, Clinical neurosciences and } \\
\text { Ophthalmology }\end{array}$ \\
\hline Keywords: & $\begin{array}{l}\text { CORD7, RIM1, Cystoid macular oedema, RAB3A-interacting molecule 1, } \\
\text { Retinitis pigmentosa }\end{array}$ \\
\hline & \\
\hline
\end{tabular}

\section{SCHOLARONE ${ }^{\text {m }}$ \\ Manuscripts}


Retinitis pigmentosa and bilateral cystoid macular oedema in a patient heterozygous for the RIM1 mutation previously associated with cone-rod dystrophy 7

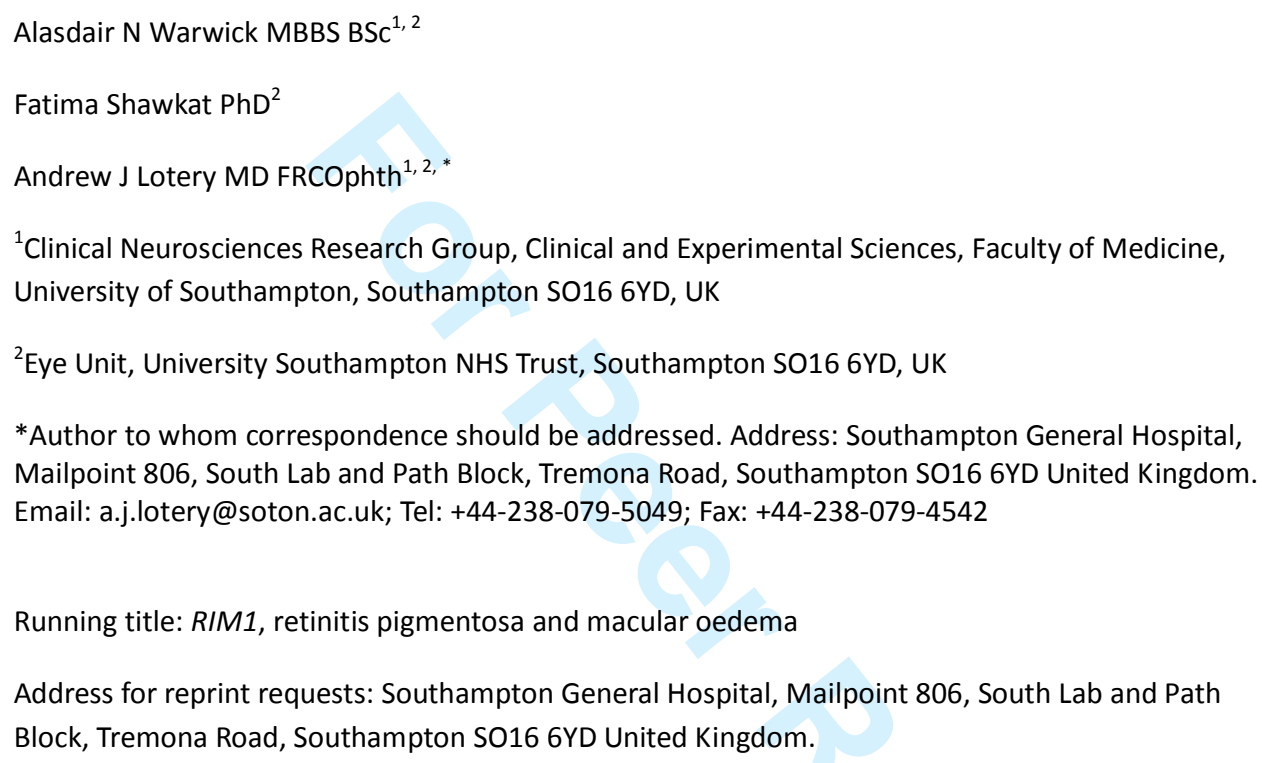


RIM1, retinitis pigmentosa and macular oedema

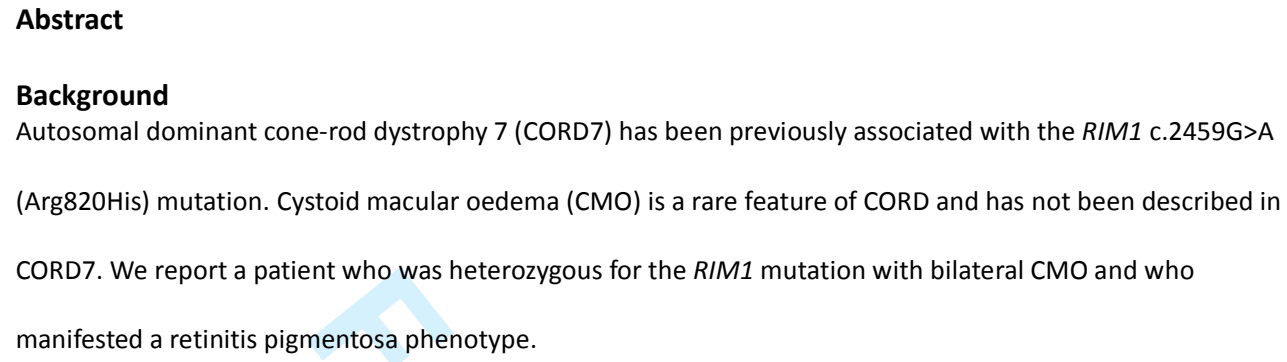


RIM1, retinitis pigmentosa and macular oedema

\begin{abstract}
Introduction
The cone-rod dystrophies (CORD) are a group of inherited retinal disorders characterised by predominant loss of cone photoreceptors with a prevalence of 1 in $40,000 .{ }^{1}$ This contrasts with the rod-cone dystrophies, also known las of which the most common type is retinitis pigmentosa, where there is primary rod photoreceptor involvement and cones are affected later with more advanced disease with a prevalence of circa 1 in $4,000 .^{2}$ Patients with CORD therefore typically present with reduced central vision and photophobia by the second decade of life, although eventually rod dysfunction also causes loss of peripheral vision and night blindness.

CORDs are highly genetically heterogeneous and inheritance may be either autosomal dominant, autosomal recessive or $\mathrm{X}$-linked. More than 30 genetic mutations have been associated with CORD to date, ${ }^{3}$ including the $\mathrm{G}$ to $\mathrm{A}$ point mutation (c.2459G $>\mathrm{A}$ ) in the Rab3A interacting molecule (RIM1) at chromosome locus 6q12-q13. ${ }^{4,5}$ This causes an Arg820His substitution in the RIM1 protein's highly conserved $\mathrm{C}_{2} \mathrm{~A}$ domain, previously associated with the autosomal dominant CORD7 phenotype. To date, the CORD7 phenotype has only been described for eight members of a four-generation, nonconsanguineous British family, none of whom developed cystoid macular oedema (CMO). ${ }^{6}$ We herein report the first case of an unrelated individual discovered to carry the Arg820His RIM1 mutation, whose clinical features were more consistent with a phenotype of retinitis pigmentosa and
\end{abstract} associated CMO.

\title{
Materials and Methods
}

The patient's medical records were reviewed. Colour fundus photographs, optical coherence tomography (OCT) scans, fundus fluorescein angiography (FFA), fundus autofluorescence, Goldmann visual field (GVF) and electrodiagnostic testing were performed. Genetic testing was performed by next generation sequencing for a panel of 176 genes known to be associated with retinal dystrophy. This next generation sequencing was outsourced and performed as a fee for service test by the Manchester Centre for Genomic Medicine, Central Manchester University Hospitals NHS Foundation 
RIM1, retinitis pigmentosa and macular oedema

(http://www.mangen.co.uk/lab-services/).

Results

A 34 year-old man presented with a five-year history of bilateral floaters and blurred vision for fine detail. He denied any photophobia, nyctalopia, loss of peripheral vision or colour blindness. He had no past ocular or past medical history. There was no significant family history, including his only brother who had no visual symptoms. No other family members were available for examination.

Visual acuity was $20 / 25$ in the right eye and $20 / 32$ in the left eye. Colour vision was not formally assessed. Intraocular pressure was $14 \mathrm{mmHg}$ bilaterally. On slit lamp examination he was noted to bilateral macular oedema, have bilateral discrete white dots peripherally at the level of the retinal pigment epithelium, a peripapillary greyish hue to the retina, and arteriolar attenuation, suggesting a possible underlying retinal dystrophy (Figure 1A). The optic disc appeared pink with clear margins. Optical coherence tomography (OCT) confirmed bilateral moderate-severe $\mathrm{CMO}$ with a central macular thickness (CMT) of $625 \mu \mathrm{m}$ and $546 \mu \mathrm{m}$ in the right and left eyes respectively.and ffFluorescein angiography (FFA) bilateral CMO furthermore revealed active leakage at both maculae (Figure 1B and $1 E(i))$.

Fundus autofluorescence scans showed blocked autofluorescence centrally, presumably due to the cystoid macular oedema, increased paracentral hypefautofluorescence and more peripheral puntcate hyper increased autofluorescence (Figure 1C). Goldmann Visual Field (GVF) tests detected mid-peripheral ring scotomas bilaterally (Figure 1D). Electrodiagnostic testing revealed significantly degraded rod and cone electroretinograms (ERG), with rods being more affected than cones (Figure 2). Interestingly, we observed a negative maximal mixed cone-rod flash ERG secondary to marked degradation of the responses, with an abnormally attenuated maximal a wave in association with an even smaller b wave. Pattern ERGs were also degraded and the $30 \mathrm{~Hz}$ flicker ERG was of diminished amplitude but normal implicit time. Subsequent genetic testing found the patient to be heterozygous for the RIM1 c.2459G>A mutation (OMIM *606629), known to cause CORD7. 
RIM1, retinitis pigmentosa and macular oedema

Topical nepafenac, ketorolac and dorzolamide, as well as oral acetazolamide were trialled for 2-4 months each with no success. However 18 months after initial presentation, the CMO had partially resolved following 4 months of topical brinzolamide therapy (Figure $1 \mathrm{E}(\mathrm{ii}))$. . Central retinal thickness as measured by OCT had improved to $509 \mu \mathrm{m}$ in the right eye and $394 \mu \mathrm{m}$ in the left eye, corresponding to reductions of $19 \%$ and $28 \%$ respectively.- with $v$ Most recent visual acuity ff was $20 / 25$ in both eyes.

\section{Discussion}

The RIM1 protein localises to the presynaptic active zone of chemical synapses in brain and retinal tissue, where it plays an important role in regulating synaptic vesicle release and presynaptic plasticity. ${ }^{7}$ RIM1 is a large multidomain protein that interacts with a host of molecules at different regions. The RIM1 Arg820His mutation associated with CORD7 affects the protein's highly conserved $\mathrm{C}_{2} \mathrm{~A}$ domain. This is thought to affect its affinity for either L-type $\mathrm{Ca}^{2+}$ channels or synaptotagmin, altering the rate of synaptic vesicle docking and fusion following a $\mathrm{Ca}^{2+}$ signal. ${ }^{5,8}$

The c.2459G>A RIM1 mutation was originally identified in a four generation, non-consanguineous British family. ${ }^{4,5}$ Michaelides subsequently provided a detailed study of the phenotype in eight members of this same family, reporting this as a cone-rod dystrophy (CORD7). ${ }^{6}$ The majority of these individuals experienced progressive worsening of central vision, nyctalopia and peripheral visual field loss between the third and fourth decades. Visual acuity ranged between 20/20 and 20/400 while fundal changes varied from mild retinal pigment epithelium changes, as with our patient, to extensive atrophy and pigmentation. AF showed decreased central hypoautofluorescence with a surrounding ring of increased hyperautofluorescence in the majority of individuals. ${ }^{6}$ The AF findings in our patient were similar, albeit more subtle. The electrodiagnostic results for our patient also resembled those found by Michaelides et al, ${ }^{6}$ showing reduced pattern, cone and rod ERGs. However our patient additionally exhibited a negative ERG, suggesting inner retinal changes secondary to a primary photoreceptor abnormality. While a negative ERG was not described by Michaelides et al, ${ }^{6}$ 
RIM1, retinitis pigmentosa and macular oedema

negative ERGs of this sort have been previously reported in CORDs. ${ }^{9,}{ }^{10}$ Perimetry showed localised central field loss and peripheral field loss in five individuals. ${ }^{6}$ By contrast our patient showed midperipheral field loss with preserved central vision on GVF testing. None of these individuals developed $\mathrm{CMO}^{6}$ Overall, the phenotype in our case more closely resembled retinitis pigmentosa with CMO rather than CORD.

Macular oedema is a common complication of retinitis pigmentosa, affecting an estimated $27 \%$ of patients. ${ }^{11}$ By contrast, CMO appears to be relatively rare in CORD. ${ }^{12-14}$ The reason for this disparity is unknown. In our case, rods were significantly affected in addition to cones, therefore perhaps rod dysfunction is required for $\mathrm{CMO}$ in retinal dystrophy. However $\mathrm{CMO}$ has been reported in a case of clinically diagnosed CORD where rod-isolated ERG responses were within normal limits. ${ }^{13}$ Treatment with carbonic anhydrase inhibitors, steroids, anti-vascular endothelial growth factor agents,

photocoagulation and vitrectomy have been trialled with variable success in retinitis pigmentosa. ${ }^{11} \underline{A}$ recent case report of cone-rod dystrophy in a child with Alstrom syndrome found complete resolution of bilateral $\mathrm{CMO}$ following one month of treatment with topical dorzolamide. ${ }^{15} \mathrm{By}$ comparison, our patient showed no response to topical dorzalamide, nepafenac, ketorolac or oral acetazolamide, and only partial resolution of CMO after 4 months of topical brinzolamide therapy.

In conclusion, we report the only phenotypic description of the RIM1 mutation outside the British family for which it was first described. This mutation has not been previously associated with either

Comment [AW12]: Reviewer 2: Recommend reviewing the paper.... Ophthalmic Genet. 2016 Feb 19:1-3. Topical carbonic anhydrase inhibitors in macular edema associated with Alström syndrome, wherein the authors report CME in a patient with CORD, and compare the findings with their patient.

Comment [AW13]: Reviewer 2: This is mere speculation. Are there any findings in their study to support this conclusion? If not suggest refraining from making this statement which is mere speculation.

Author response: as the clinical picture in our case more closely resembled retinitis pigmentosa than CORD, we have tried to provide speculative reasons which may explain this discrepancy with the earlier report by Michaelides et al.

Furthermore, this last sentence was added as per the previous suggestion from reviewer 3 . We have therefore kept this in the manuscript. 
RIM1, retinitis pigmentosa and macular oedema

\section{Declaration of interest}

The authors report no conflicts of interest. The authors alone are responsible for the content and writing of the paper.

\section{Acknowledgements}

None.

\section{Figure legends}

\section{Figure 1}

Title: Clinical imaging and Goldmann visual field findings

Legend: Fundus photographs (A) showing cystoid macular oedema, arteriolar attenuation, a peripapillary greyish hue to the retina, as well as scattered areas of nummular pigmentation and mottling of the peripheral retinal pigment epithelium with scattered white dots peripherally. The optic disc appears pink with clear margins. Wide field fluorescein angiography (B) demonstrates bilateral early mid-peripheral hyperfluorescence indicating leakage. Fundus autofluorescence imaging (C) showing blocked autofluorescence centrally, presumably due to the cystoid macular oedema, increased paracentral hyperautofluorescence and more peripheral puntcate increased hyperautofluorescence. Goldmann visual fields (D) reveals bilateral mid-peripheral ring scotomas. Optical coherence tomography (OCT) scans (E) showing bilateral cystoid macular oedema at presentation (i) and partial improvement at 18 months follow-up (ii). Central macular thickness measured with Heidelberg OCT reduced over 18 months from $625-\mu \mathrm{m}$ to $509 \mu \mathrm{m}$ OD-_and from 546 $\mu \mathrm{m}$ OS, to $509 \mu \mathrm{m}$ OD and to $394-\mu \mathrm{m}$ OS.

Figure 2

Title: Electrodiagnostic testing results

Legend: Electrophysiological tracings from the patient with normal traces for comparison. Pattern ERG (PERG), maximal mixed cone-rod flash ERGs and independent cone and rod ERGs were all markedly degraded, with rods being more affected than cones. The mixed cone-rod flash ERG was of negative configuration with an abnormal maximal a wave in association with an even smaller $b$ wave, suggesting a primary photoreceptor abnormality with inner retinal changes secondary to photoreceptor dysfunction. The $30 \mathrm{~Hz}$ flicker ERG was of diminished amplitude but normal implicit time. 
RIM1, retinitis pigmentosa and macular oedema

\section{References}

1. Hamel CP. Cone rod dystrophies. Orphanet J. Rare Dis. 2007; 2: 7.

2. Hartong DT, Berson EL, Dryja TP. Retinitis pigmentosa. Lancet 2006; 368: 1795-1809.

3. Boulanger-Scemama E, El Shamieh S, Demontant V, Condroyer C, Antonio A, Michiels C et al. Nextgeneration sequencing applied to a large French cone and cone-rod dystrophy cohort: mutation spectrum and new genotype-phenotype correlation. Orphanet J. Rare Dis. 2015; 10: 85-015-0300-3.

4. Kelsell RE, Gregory-Evans K, Gregory-Evans CY, Holder GE, Jay MR, Weber BH et al. Localization of a gene (CORD7) for a dominant cone-rod dystrophy to chromosome 6q. Am. J. Hum. Genet. 1998; 63: 274-279.

5. Johnson S, Halford S, Morris AG, Patel RJ, Wilkie SE, Hardcastle AJ et al. Genomic organisation and alternative splicing of human RIM1, a gene implicated in autosomal dominant cone-rod dystrophy (CORD7). Genomics 2003; 81: 304-314.

6. Michaelides M, Holder GE, Hunt DM, Fitzke FW, Bird AC, Moore AT. A detailed study of the phenotype of an autosomal dominant cone-rod dystrophy (CORD7) associated with mutation in the gene for RIM1. Br. J. Ophthalmol. 2005; 89: 198-206.

7. Mittelstaedt T, Alvarez-Baron E, Schoch S. RIM proteins and their role in synapse function. Biol. Chem. 2010; 391: 599-606.

8. Miki T, Kiyonaka S, Uriu Y, De Waard M, Wakamori M, Beedle AM et al. Mutation associated with an autosomal dominant cone-rod dystrophy CORD7 modifies RIM1-mediated modulation of voltagedependent Ca2+ channels. Channels (Austin) 2007; 1: 144-147.

9. Fujii N, Shiono T, Wada Y, Nakazawa M, Tamai M, Yamada N. Autosomal dominant cone-rod dystrophy with negative electroretinogram. Br. J. Ophthalmol. 1995; 79: 916-921.

10. Holopigian K, Greenstein VC, Seiple W, Hood DC, Carr RE. Rod and cone photoreceptor function in patients with cone dystrophy. Invest. Ophthalmol. Vis. Sci. 2004; 45: 275-281.

11. Makiyama Y, Oishi A, Otani A, Ogino K, Nakagawa S, Kurimoto M et al. Prevalence and spatial distribution of cystoid spaces in retinitis pigmentosa: investigation with spectral domain optical coherence tomography. Retina 2014; 34: 981-988.

12. Emfietzoglou I, Grigoropoulos V, Nikolaidis P, Theodossiadis G, Rouvas A, Theodossiadis P. Optical coherence tomography findings in a case of cone-rod dystrophy. Ophthalmic Surg. Lasers Imaging 2010; 41 Online: e1-3.

13. Gelman SK, Gorin MB. Significant macular edema in a patient with cone dystrophy and improvement with acetazolamide treatment. Retin. Cases Brief Rep. 2014; 8: 300-305.

14. Salvatore S, Genead MA, Fishman GA. The prevalence of macular cysts in patients with clinical cone-rod dystrophy determined by spectral-domain optical coherence tomography. Ophthalmic Genet. 2014; 35: 47-50.

15. Larranaga-Fragoso P, Pastora N, Bravo-Ljubetic L, Peralta J, Abelairas-Gomez J. Topical carbonic anhydrase inhibitors in macular edema associated with Alstrom syndrome. Ophthalmic Genet. 2016; 1-3. 


\section{Page 9 of 10 Right eye}

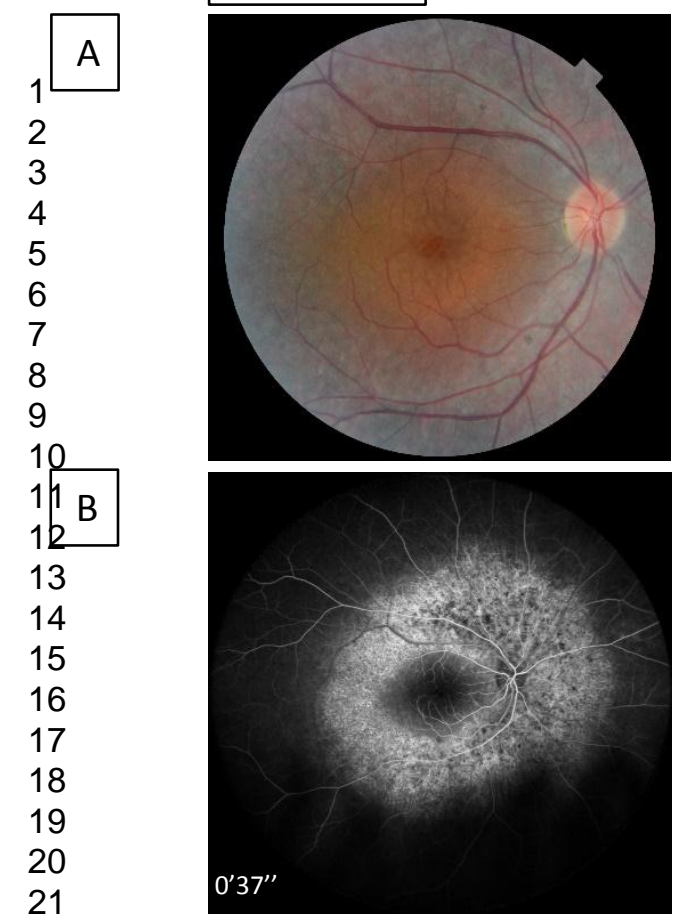

22

23

24

25

26

27

28

29

30

31

32

$3 \beta$

34

36

37

38

39

40

41

42

43

44

45 ᄃ

46

47

48

49

50

51

52

53

54

55 5 5
5 58

59

60


(ii)
Ophthalmic Geneticsft eye
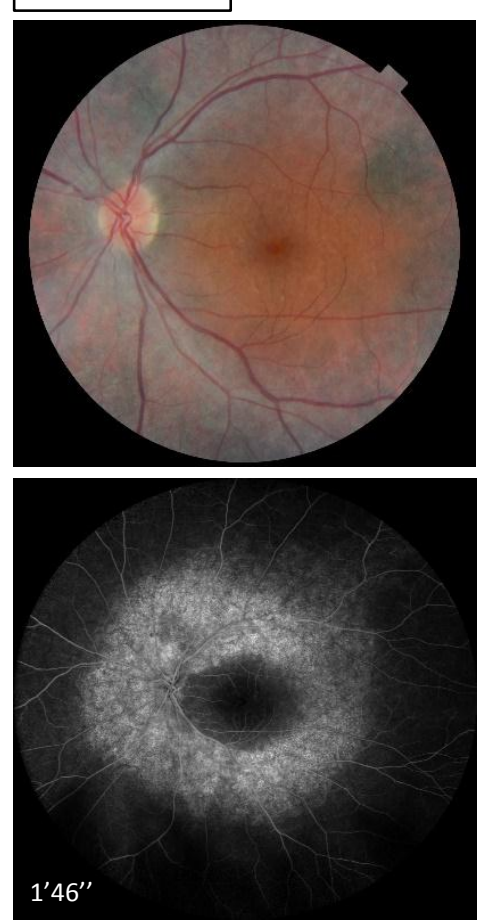

4'57'

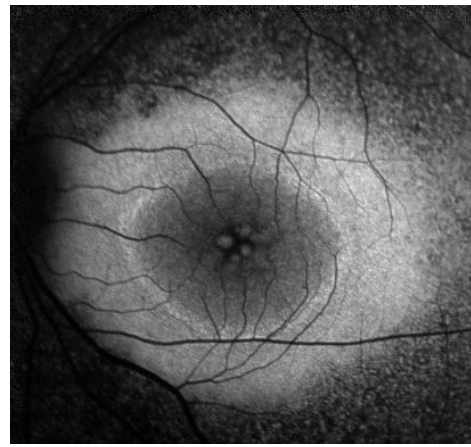

발 sagh he
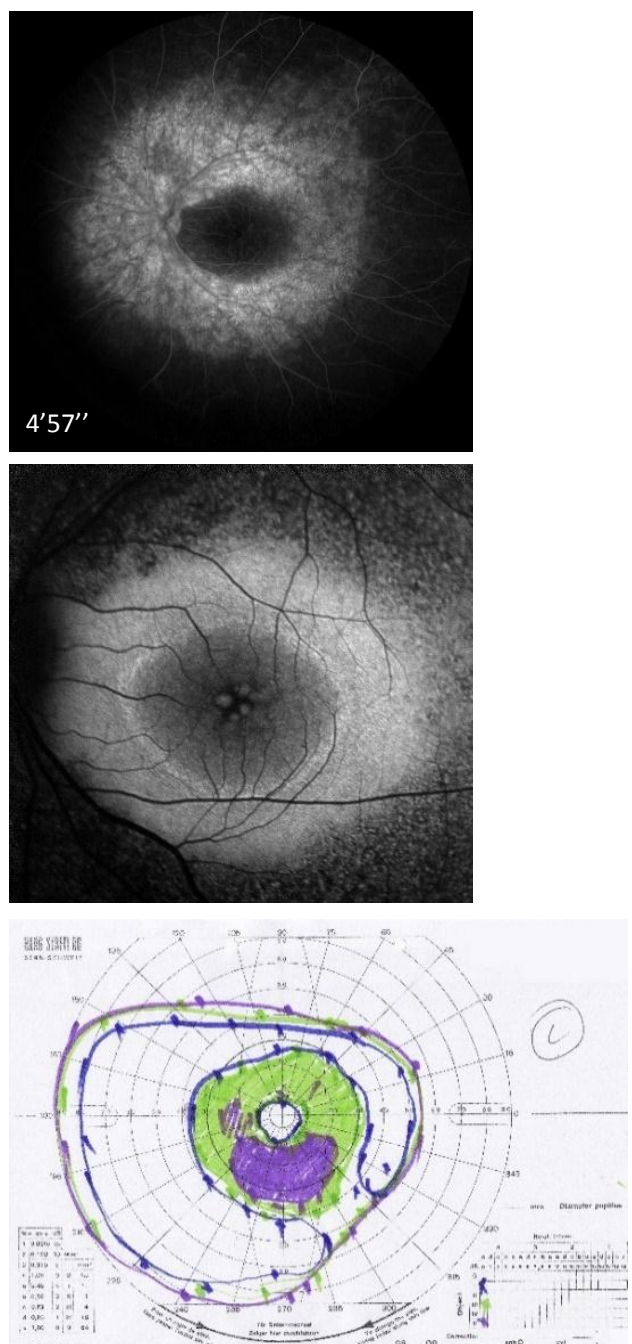

(i)
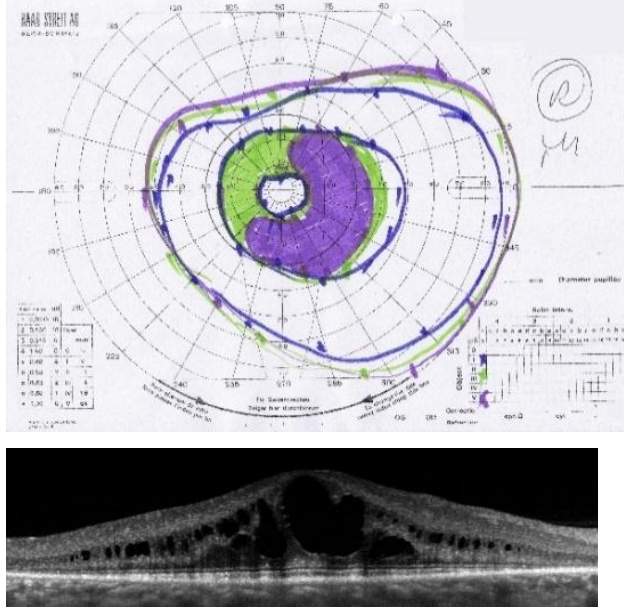

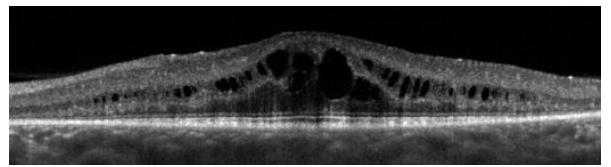

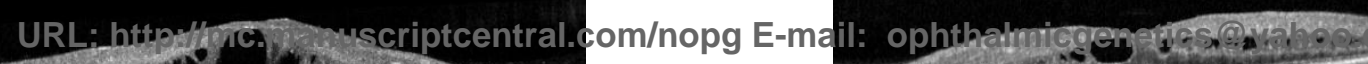

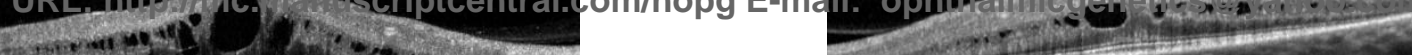

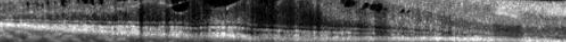



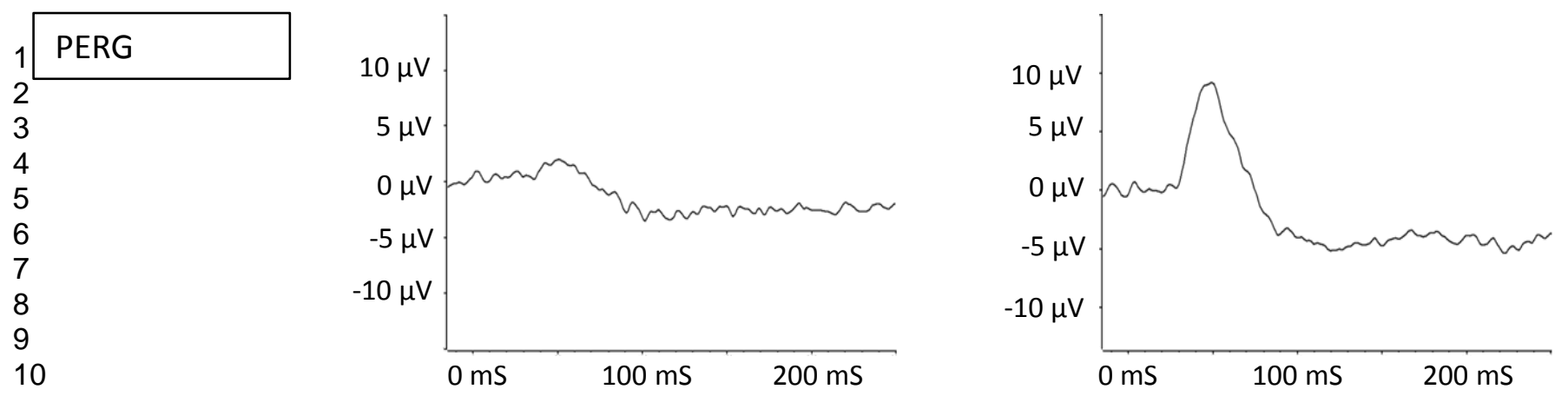

11
12
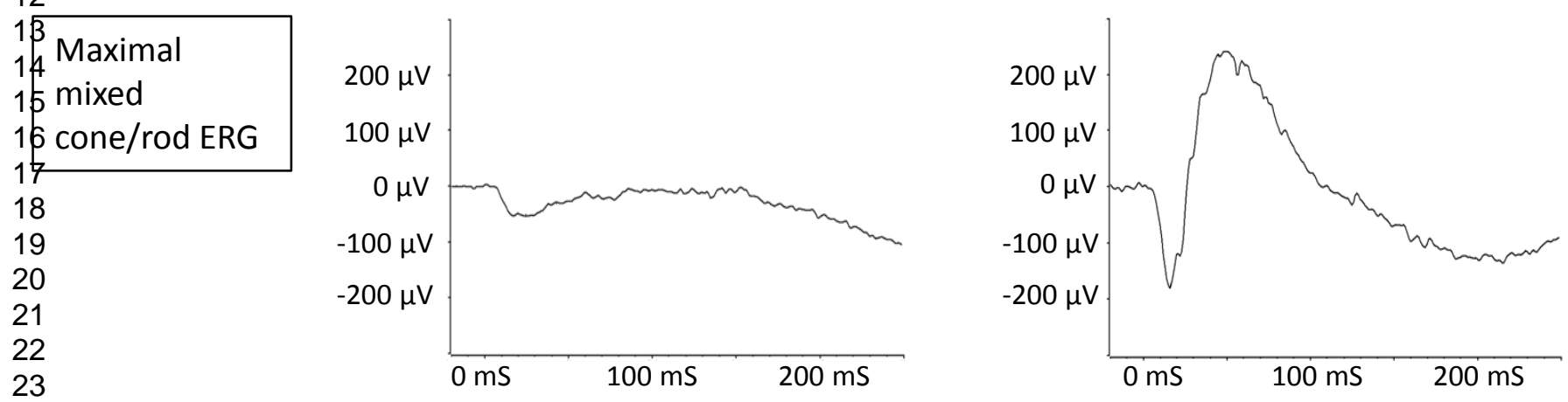

Scotopic rod ERG

29

30

31

32

33

34

35

36

37

\section{8}

39 Photopic cone 4 ERG

44

42

43

44

45

46

47

48

49

50
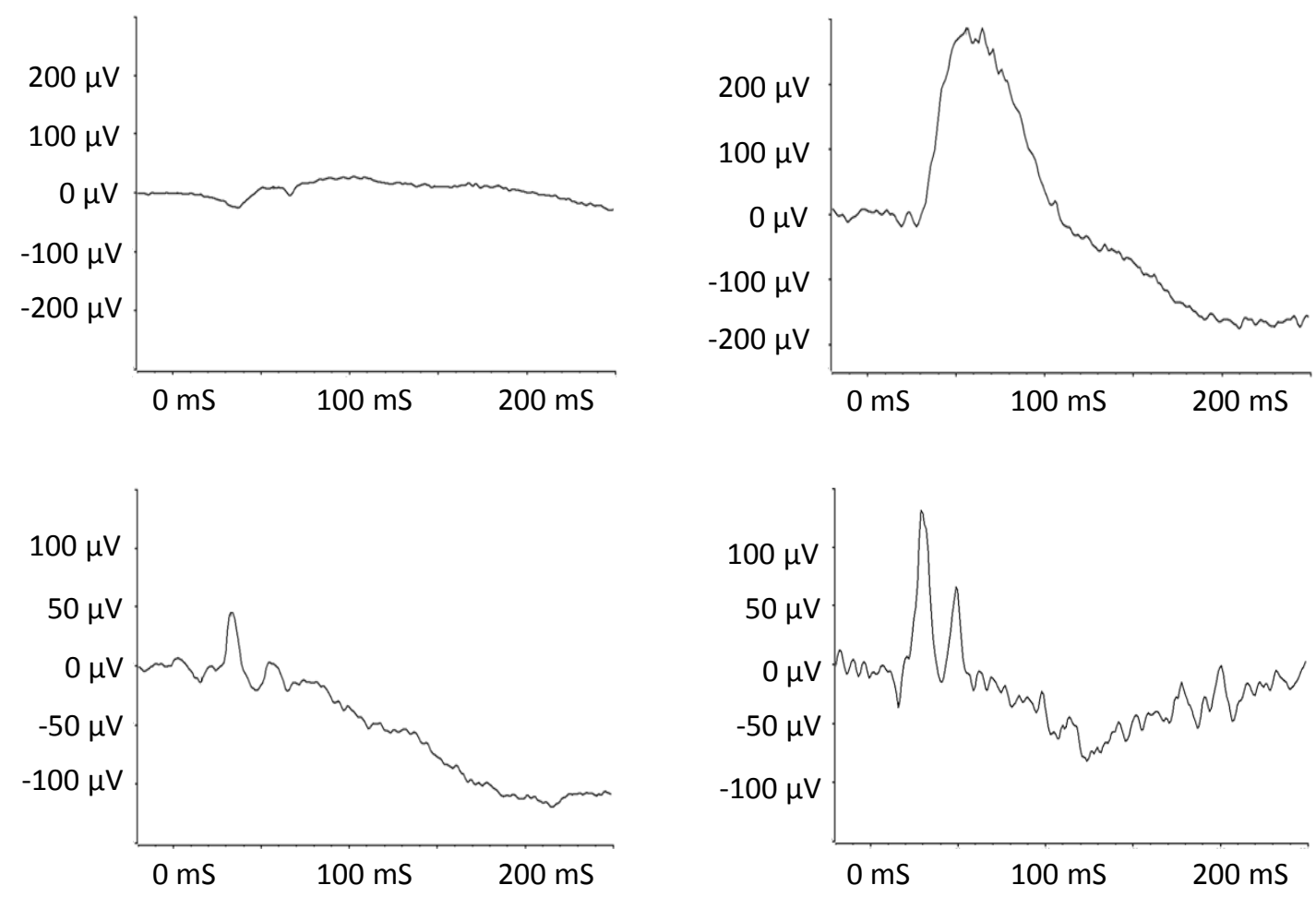

\section{$30 \mathrm{~Hz}$ flicker}

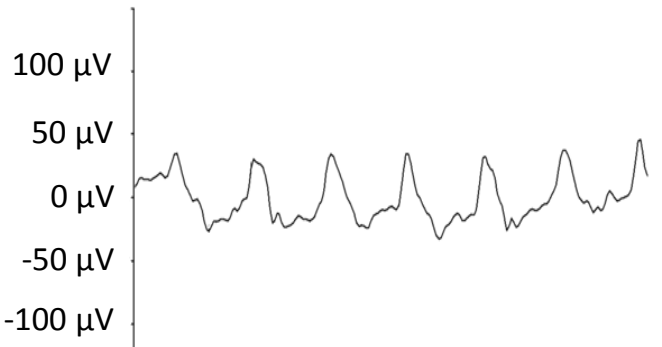

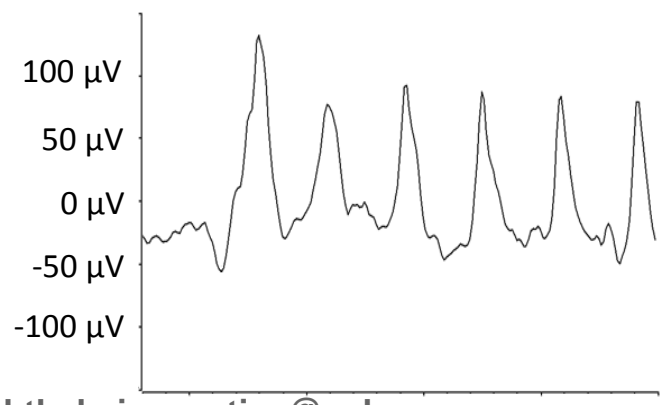

